\title{
A variety of exact solutions for fractional (2+1)-dimensional Heisenberg ferromagnetic spin chain in the semiclassical limit
}

\author{
J. Akhtar ${ }^{a}$, K. U. Tariq ${ }^{a}$, M. M. A. Khater ${ }^{b, c}$, A. Houwe ${ }^{d}$, and M. Inc ${ }^{e, f, g, *}$ \\ ${ }^{a}$ Department of Mathematics, Mirpur University of Science and Technology, \\ Mirpur-10250 (AJK), Pakistan. \\ ${ }^{b}$ Department of Mathematics, Faculty of Science, Jiangsu University, 212013, Zhenjiang, China. \\ ${ }^{c}$ Department of Mathematics, Obour High Institute for Engineering and Technology, 11828, Cairo, Egypt. \\ e-mail: mostafa.khater2024@yahoo.com \\ ${ }^{d}$ Department of Physics, Faculty of Science, University of Maroua, P.O. Box 814 Cameroon \\ ${ }^{e}$ Biruni University, Department of Computer Engineering, Istanbul, Turkey \\ ${ }^{f}$ Firat University, Science Faculty, Department of Mathematics, 23119 Elazig, Turkey. \\ ${ }^{g}$ Department of Medical Research, China Medical University Hospital, China Medical University, Taichung, Taiwan. \\ *e-mail:minc@firat.edu.tr
}

Received 16 December 2020; accepted 22 January 2021

\begin{abstract}
This paper investigates exact voyaging $(2+1)$ dimensional Heisenberg ferromagnetic spin chain solutions with conformable fractional derivatives, an important family of nonlinear equations from Schrödinger (NLSE) for the construction of hyperbolic, trigonometric and complex function solutions. The detailed rational sine-cosine system and rational sinh-cosh system were used to locate dim, special and periodic wave solutions successfully. These findings suggest that the proposed approaches may be useful to investigate a range of solutions inside a repository of applied sciences and engineering, with success, quality, and trust. In addition, graphical representations and physical expresses of such solutions are represented by a set of the required values of the parameters involved. The methods are essentially adequate and can be extended to different dynamic models that create the nonlinear processes in today's research.
\end{abstract}

Keywords: Heisenberg ferromagnetic spin chain; conformable fraction derivative; extended rational trigonometric method; exact solutions.

PACS: 02.30.Jr; 05.45.Yv; 47.10.A-; 47.35.Fg

DOI: https://doi.org/10.31349/RevMexFis.67.040701

\section{Introduction}

Over the last few years, nonlinear Schrödinger's equations (NLSEs) have attracted much attention in the field of research due to their numerous fascinating behaviour and countless characteristics. A large variety of these equations are utilized to describe important phenomena in different scientific fields like, plasma physics [1,2], condensed matter physics [3], convective fluids [5], optical fibers [6,7], solid state physics $[8,9]$, hydrodynamic [10], water waves [11] and many other branches of engineering [12-14]. In past years, to find the exact solutions of NLSEs many powerful technique have been developed such as, the inverse scattering transformation [15], the homotopy perturbation method [16,17], the Darboux transformation method $[18,19]$, the Sine-Gordon expansion method [20], Bernoulli sub-equation method [21], the modified auxiliary equation mapping method [22,23], the Riccati equation mapping method [4], the extended sinh-Gordon equation expansion method [24],the modify extended direct algebraic method [25].

The Heisenberg models of ferromagnetic spin chains with various magnetic reactions in the classical and semiclassical limits have been related with nonlinear evaluation equations (NLEEs). The nonlinear spin chain have wide range of applications in magnetic materials such as, sensors [26], microwave, date storage devices, communication system [27], signal processing devices and quantum computing. In this article, we have successfully investigated a variety of exact travelling wave solutions by employing extended rational trigonometric methods to construct the hyperbolic, trigonometric and complex function solutions moreover classify as dark, singular and periodic wave solutions. To study $(2+1)-$ dimensional Heisenberg ferromagnetic spin chains (HFSC) model of the the form $[28,29]$.

$$
i \Psi_{t}+\mu \Psi_{x x}+\lambda \Psi_{y y}+\eta \Psi_{x y}-\delta|\Psi|^{2} \Psi=0,
$$

here, $\Psi$ is coherent amplitude. Hashemi transform it into fractional form:

$$
\begin{aligned}
i D_{t}^{\alpha}(\Psi)+\mu \Psi_{x x}+\lambda \Psi_{y y}+\eta \Psi_{x y}-\delta|\Psi|^{2} \Psi & =0, \\
i & =\sqrt{-1}
\end{aligned}
$$

where $\Psi=\Psi(x, y, t)$ is the complex valued function of Heisenberg ferromagnetic spin chain, $x$ and $y$ are representing scaled spatial and $\mathrm{t}$ is the time coordinates respectively.

In recent times, a large number of scientists and researchers have been attracted to HFSC models due to their significant and fascinating characteristics for construction of different types of exact solutions in NPDEs. $D_{t}^{\alpha}(\Psi)$ is the conformable fraction derivative of $\Psi$ of order $\alpha$. Nowadays, the field of conformable fractional derivative become one of the most important and interesting field for scientists because 
of its uses nonlinear sciences suck as, fluid mechanics, chemical and biological processes. In literature, there are so many definitions which of them are, Riemann-Liouville [30,31], Atangana-Baleanu derivative in Caputo sense [32], Caputoa and Grunwald-Letnikov [33-35].

The remaining paper is arranged as fellows: In Sec. 2 some sliton solutions to the HFSC model have been presented. The physical significance and graphical representation is presented in Sec. 3. In Sec. 4 finally the concluding remarks and behaviour of solution have been discussed.

\section{Mathematical analysis}

In this section, to obtain the exact solutions of Eq. (2) by applying following conformable fractional derivative [36]

$$
D_{t}^{\alpha}(\Psi(t))=\lim _{\epsilon \rightarrow 0} \frac{\Psi\left(t+\epsilon t^{t-\alpha}\right)-\Psi(t)}{\epsilon} .
$$

By this definition and following complex travelling wave transformation

$$
\Phi(x, y, t)=\Psi(s) e^{i \Theta(x, y, t)},
$$

where

$$
\begin{aligned}
& s=\alpha_{1} x+\alpha_{2} y-\omega \frac{t^{\alpha}}{\alpha}, \\
& \Theta=-\beta_{1} x-\beta_{2} y+\rho \frac{t^{\alpha}}{\alpha}+\theta .
\end{aligned}
$$

Putting Eq. (5) into Eq. (2), we obtain

$$
\begin{aligned}
& \left(\mu \alpha_{1}^{2}+\eta \alpha_{1} \alpha_{2}+\lambda \alpha_{2}^{2}\right) \Psi^{\prime \prime}(s) \\
& -\left(\rho+\mu \beta_{1}^{2}+\eta \beta_{1} \beta_{2}+\lambda \beta_{2}^{2}\right) \Psi(s)-\delta \Psi(s)^{3}=0,
\end{aligned}
$$

where $\alpha_{1}, \alpha_{2}, \beta_{1}$ and $\beta_{2}$ real constants and are center of phase. where $\Theta$ represents the phase component, $\rho$ is the velocity and $\omega$ is the frequency respectively.

\subsection{Applications of the extended rational sine-cosine method}

Assume that Eq. (6) has the solution of the form:

$$
\Psi(s)=\frac{A_{0} \sin (\nu s)}{A_{2}+A_{1} \cos (\nu s)}, \quad \cos (\nu s) \neq-\frac{A_{2}}{A_{1}},
$$

or of the form

$$
\Psi(s)=\frac{A_{0} \cos (\nu s)}{A_{2}+A_{1} \sin (\nu s)}, \quad \sin (\nu s) \neq-\frac{A_{2}}{A_{1}},
$$

where $A_{0}, A_{1}$ and $A_{2}$ are parameters that will be determined and $\nu$ represents wave number.

Family I

Now, substituting Eq. (7) into Eq. (6) and then setting each coefficients of all terms of $\cos ^{m}(\nu s)$ or $\sin ^{m}(\nu s)$ to zero, yields a system of algebraic equations. Then we obtain system of algebraic equations involving parameters $A_{0}, A_{1}, A_{2}, \mu, \nu, \lambda, \delta, \rho$.

$$
\begin{array}{r}
-\delta A_{0}^{2}+A_{1}^{2}\left(\rho+\mu \beta_{1}^{2}+\eta \beta_{1} \beta_{2}+\lambda \beta_{2}^{2}\right)=0 \\
A_{1} A_{2}\left[-\mu \nu^{2} \alpha_{1}^{2}-\eta \nu^{2} \alpha_{1} \alpha_{2}-\lambda \nu^{2} \alpha_{2}^{2}+2\left(\rho+\mu \beta_{1}^{2}+\eta \beta_{1} \beta_{2}+\lambda \beta_{2}^{2}\right)\right]=0 \\
\delta A_{0}^{2}-2 \nu^{2} A_{1}^{2}\left(\mu \alpha_{1}^{2}+\eta \alpha_{1} \alpha_{2}+\lambda \alpha_{2}^{2}\right)+A_{2}^{2}\left(\rho+\mu \nu^{2} \alpha_{1}^{2}+\eta \nu^{2} \alpha_{1} \alpha_{2}+\lambda \nu^{2} \alpha_{2}^{2}+\mu \beta_{1}^{2}+\eta \beta_{1} \beta_{2}+\lambda \beta_{2}^{2}\right)=0 .
\end{array}
$$

Solving this system, we yields the following set:

Case I:

$$
A_{0}= \pm A_{1} \sqrt{\frac{\rho+\mu \beta_{1}^{2}+\eta \beta_{1} \beta_{2}+\lambda \beta_{2}^{2}}{\delta}}, \quad A_{1}= \pm A_{2}, \quad \nu= \pm \sqrt{\frac{2\left(\rho+\mu \beta_{1}^{2}+\eta \beta_{1} \beta_{2}+\lambda \beta_{2}^{2}\right)}{\mu \alpha_{1}^{2}+\eta \alpha_{1} \alpha_{2}+\lambda \alpha_{2}^{2}}}
$$

Substituting these results into Eq. (5) by using Eq. (7), we have

$$
\begin{aligned}
& \Phi_{1,1}(x, y, t)=\sqrt{\frac{\rho+\mu \beta_{1}^{2}+\eta \beta_{1} \beta_{2}+\lambda \beta_{2}^{2}}{\delta}}\left(\frac{\sin \left[\sqrt{\frac{2\left(\rho+\mu \beta_{1}^{2}+\eta \beta_{1} \beta_{2}+\lambda \beta_{2}^{2}\right)}{\mu \alpha_{1}^{2}+\eta \alpha_{1} \alpha_{2}+\lambda \alpha_{2}^{2}}}(s)\right]}{1+\cos \left[\sqrt{\frac{2\left(\rho+\mu \beta_{1}^{2}+\eta \beta_{1} \beta_{2}+\lambda \beta_{2}^{2}\right)}{\mu \alpha_{1}^{2}+\eta \alpha_{1} \alpha_{2}+\lambda \alpha_{2}^{2}}}(s)\right]}\right) e^{i\left\{-\beta_{1} x-\beta_{2} y+\rho \frac{t^{\alpha}}{\alpha}+\theta\right\}} . \\
& \Phi_{1,2}(x, y, t)=-\sqrt{\frac{\rho+\mu \beta_{1}^{2}+\eta \beta_{1} \beta_{2}+\lambda \beta_{2}^{2}}{\delta}}\left(\frac{\sin \left[\sqrt{\frac{2\left(\rho+\mu \beta_{1}^{2}+\eta \beta_{1} \beta_{2}+\lambda \beta_{2}^{2}\right)}{\mu \alpha_{1}^{2}+\eta \alpha_{1} \alpha_{2}+\lambda \alpha_{2}^{2}}}(s)\right]}{1+\cos \left[\sqrt{\frac{2\left(\rho+\mu \beta_{1}^{2}+\eta \beta_{1} \beta_{2}+\lambda \beta_{2}^{2}\right)}{\mu \alpha_{1}^{2}+\eta \alpha_{1} \alpha_{2}+\lambda \alpha_{2}^{2}}}(s)\right]}\right] e^{i\left\{-\beta_{1} x-\beta_{2} y+\rho \frac{t^{\alpha}}{\alpha}+\theta\right\}} .
\end{aligned}
$$




$$
\begin{aligned}
\Phi_{1,3}(x, y, t) & =\sqrt{\frac{\rho+\mu \beta_{1}^{2}+\eta \beta_{1} \beta_{2}+\lambda \beta_{2}^{2}}{\delta}}\left(\frac{\sin \left[\sqrt{\frac{2\left(\rho+\mu \beta_{1}^{2}+\eta \beta_{1} \beta_{2}+\lambda \beta_{2}^{2}\right)}{\mu \alpha_{1}^{2}+\eta \alpha_{1} \alpha_{2}+\lambda \alpha_{2}^{2}}}(s)\right]}{\left.1-\cos \left[\sqrt{\frac{2\left(\rho+\mu \beta_{1}^{2}+\eta \beta_{1} \beta_{2}+\lambda \beta_{2}^{2}\right)}{\mu \alpha_{1}^{2}+\eta \alpha_{1} \alpha_{2}+\lambda \alpha_{2}^{2}}}(s)\right]\right)}\right) e^{i\left\{-\beta_{1} x-\beta_{2} y+\rho \frac{t^{\alpha}}{\alpha}+\theta\right\}} . \\
\Phi_{1,4}(x, y, t) & =-\sqrt{\frac{\rho+\mu \beta_{1}^{2}+\eta \beta_{1} \beta_{2}+\lambda \beta_{2}^{2}}{\delta}}\left(\frac{\sin \left[\sqrt{\frac{2\left(\rho+\mu \beta_{1}^{2}+\eta \beta_{1} \beta_{2}+\lambda \beta_{2}^{2}\right)}{\mu \alpha_{1}^{2}+\eta \alpha_{1} \alpha_{2}+\lambda \alpha_{2}^{2}}}(s)\right]}{1-\cos \left[\sqrt{\frac{2\left(\rho+\mu \beta_{1}^{2}+\eta \beta_{1} \beta_{2}+\lambda \beta_{2}^{2}\right)}{\mu \alpha_{1}^{2}+\eta \alpha_{1} \alpha_{2}+\lambda \alpha_{2}^{2}}}(s)\right]} e^{i\left\{-\beta_{1} x-\beta_{2} y+\rho \frac{t^{\alpha}}{\alpha}+\theta\right\}} .\right.
\end{aligned}
$$

Case II:

$$
A_{0}= \pm A_{1} \sqrt{\frac{\rho+\mu \beta_{1}^{2}+\eta \beta_{1} \beta_{2}+\lambda \beta_{2}^{2}}{\delta}}, \quad A_{2}=0, \quad \nu= \pm \sqrt{\frac{\rho+\mu \beta_{1}^{2}+\eta \beta_{1} \beta_{2}+\lambda \beta_{2}^{2}}{2\left(\mu \alpha_{1}^{2}+\eta \alpha_{1} \alpha_{2}+\lambda \alpha_{2}^{2}\right)}} .
$$

Again, substituting these results into Eq. (5) by using Eq. (7), we obtain

$$
\begin{aligned}
& \Phi_{1,5}(x, y, t)=\sqrt{\frac{\rho+\mu \beta_{1}^{2}+\eta \beta_{1} \beta_{2}+\lambda \beta_{2}^{2}}{\delta}}\left(\tan \left[\sqrt{\frac{\rho+\mu \beta_{1}^{2}+\eta \beta_{1} \beta_{2}+\lambda \beta_{2}^{2}}{2\left(\mu \alpha_{1}^{2}+\eta \alpha_{1} \alpha_{2}+\lambda \alpha_{2}^{2}\right)}}(s)\right]\right) e^{i\left\{-\beta_{1} x-\beta_{2} y+\rho \frac{t^{\alpha}}{\alpha}+\theta\right\}} . \\
& \Phi_{1,6}(x, y, t)=-\sqrt{\frac{\rho+\mu \beta_{1}^{2}+\eta \beta_{1} \beta_{2}+\lambda \beta_{2}^{2}}{\delta}}\left(\tan \left[\sqrt{\frac{\rho+\mu \beta_{1}^{2}+\eta \beta_{1} \beta_{2}+\lambda \beta_{2}^{2}}{2\left(\mu \alpha_{1}^{2}+\eta \alpha_{1} \alpha_{2}+\lambda \alpha_{2}^{2}\right)}}(s)\right]\right) e^{i\left\{-\beta_{1} x-\beta_{2} y+\rho \frac{t^{\alpha}}{\alpha}+\theta\right\}},
\end{aligned}
$$

provided that $\left(\rho+\mu \beta_{1}^{2}+\eta \beta_{1} \beta_{2}+\lambda \beta_{2}^{2}\right)\left(\mu \alpha_{1}^{2}+\eta \alpha_{1} \alpha_{2}+\lambda \alpha_{2}^{2}\right)>0$.

Family II

Again, substituting Eq. (8) into Eq. (6) and then setting each coefficients of all terms of $\sin ^{m}(\nu s)$ or $\cos ^{m}(\nu s)$ to zero, yields a system of algebraic equations. Then we obtain system of algebraic equations involving parameters $A_{0}, A_{1}, A_{2}, \mu, \nu$, $\lambda, \delta, \rho$.

$$
\begin{aligned}
-\delta A_{0}^{2}+A_{1}^{2}\left(\rho+\mu \beta_{1}^{2}+\eta \beta_{1} \beta_{2}+\lambda \beta_{2}^{2}\right) & =0, \\
A_{1} A_{2}\left[-\mu \nu^{2} \alpha_{1}^{2}-\eta \nu^{2} \alpha_{1} \alpha_{2}-\lambda \nu^{2} \alpha_{2}^{2}+2\left(\rho+\mu \beta_{1}^{2}+\eta \beta_{1} \beta_{2}+\lambda \beta_{2}^{2}\right)\right] & =0, \\
\delta A_{0}^{2}-2 \nu^{2} A_{1}^{2}\left(\mu \alpha_{1}^{2}+\eta \alpha_{1} \alpha_{2}+\lambda \alpha_{2}^{2}\right)+A_{2}^{2}\left(\rho+\mu \nu^{2} \alpha_{1}^{2}+\eta \nu^{2} \alpha_{1} \alpha_{2}+\lambda \nu^{2} \alpha_{2}^{2}+\mu \beta_{1}^{2}+\eta \beta_{1} \beta_{2}+\lambda \beta_{2}^{2}\right) & =0 .
\end{aligned}
$$

This system, gives the following set of solutions:

Case I:

$$
A_{0}= \pm A_{1} \sqrt{\frac{\rho+\mu \beta_{1}^{2}+\eta \beta_{1} \beta_{2}+\lambda \beta_{2}^{2}}{\delta}}, \quad A_{1}= \pm A_{2}, \quad \nu= \pm \sqrt{\frac{2\left(\rho+\mu \beta_{1}^{2}+\eta \beta_{1} \beta_{2}+\lambda \beta_{2}^{2}\right)}{\mu \alpha_{1}^{2}+\eta \alpha_{1} \alpha_{2}+\lambda \alpha_{2}^{2}}} .
$$

Substituting these results into Eq. (5) by using Eq. (8), we have

$$
\Phi_{2,1}(x, y, t)=\sqrt{\frac{\rho+\mu \beta_{1}^{2}+\eta \beta_{1} \beta_{2}+\lambda \beta_{2}^{2}}{\delta}}\left(\frac{\cos \left[\sqrt{\frac{2\left(\rho+\mu \beta_{1}^{2}+\eta \beta_{1} \beta_{2}+\lambda \beta_{2}^{2}\right)}{\mu \alpha_{1}^{2}+\eta \alpha_{1} \alpha_{2}+\lambda \alpha_{2}^{2}}}(s)\right]}{1+\sin \left[\sqrt{\frac{2\left(\rho+\mu \beta_{1}^{2}+\eta \beta_{1} \beta_{2}+\lambda \beta_{2}^{2}\right)}{\mu \alpha_{1}^{2}+\eta \alpha_{1} \alpha_{2}+\lambda \alpha_{2}^{2}}}(s)\right]}\right) e^{i\left\{-\beta_{1} x-\beta_{2} y+\rho \frac{t^{\alpha}}{\alpha}+\theta\right\}} .
$$




$$
\begin{aligned}
& \Phi_{2,2}(x, y, t)=-\sqrt{\frac{\rho+\mu \beta_{1}^{2}+\eta \beta_{1} \beta_{2}+\lambda \beta_{2}^{2}}{\delta}}\left(\frac{\cos \left[\sqrt{\frac{2\left(\rho+\mu \beta_{1}^{2}+\eta \beta_{1} \beta_{2}+\lambda \beta_{2}^{2}\right)}{\mu \alpha_{1}^{2}+\eta \alpha_{1} \alpha_{2}+\lambda \alpha_{2}^{2}}}(s)\right]}{1+\sin \left[\sqrt{\frac{2\left(\rho+\mu \beta_{1}^{2}+\eta \beta_{1} \beta_{2}+\lambda \beta_{2}^{2}\right)}{\mu \alpha_{1}^{2}+\eta \alpha_{1} \alpha_{2}+\lambda \alpha_{2}^{2}}}(s)\right]}\right) e^{i\left\{-\beta_{1} x-\beta_{2} y+\rho \frac{t^{\alpha}}{\alpha}+\theta\right\}} . \\
& \Phi_{2,3}(x, y, t)=\sqrt{\frac{\rho+\mu \beta_{1}^{2}+\eta \beta_{1} \beta_{2}+\lambda \beta_{2}^{2}}{\delta}}\left(\frac{\cos \left[\sqrt{\frac{2\left(\rho+\mu \beta_{1}^{2}+\eta \beta_{1} \beta_{2}+\lambda \beta_{2}^{2}\right)}{\mu \alpha_{1}^{2}+\eta \alpha_{1} \alpha_{2}+\lambda \alpha_{2}^{2}}}(s)\right]}{1-\sin \left[\sqrt{\frac{2\left(\rho+\mu \beta_{1}^{2}+\eta \beta_{1} \beta_{2}+\lambda \beta_{2}^{2}\right)}{\mu \alpha_{1}^{2}+\eta \alpha_{1} \alpha_{2}+\lambda \alpha_{2}^{2}}}(s)\right]}\right) e^{i\left\{-\beta_{1} x-\beta_{2} y+\rho \frac{t^{\alpha}}{\alpha}+\theta\right\}} . \\
& \Phi_{2,4}(x, y, t)=-\sqrt{\frac{\rho+\mu \beta_{1}^{2}+\eta \beta_{1} \beta_{2}+\lambda \beta_{2}^{2}}{\delta}}\left(\frac{\cos \left[\sqrt{\frac{2\left(\rho+\mu \beta_{1}^{2}+\eta \beta_{1} \beta_{2}+\lambda \beta_{2}^{2}\right)}{\mu \alpha_{1}^{2}+\eta \alpha_{1} \alpha_{2}+\lambda \alpha_{2}^{2}}}(s)\right]}{1-\sin \left[\sqrt{\frac{2\left(\rho+\mu \beta_{1}^{2}+\eta \beta_{1} \beta_{2}+\lambda \beta_{2}^{2}\right)}{\mu \alpha_{1}^{2}+\eta \alpha_{1} \alpha_{2}+\lambda \alpha_{2}^{2}}}(s)\right]}\right) e^{i\left\{-\beta_{1} x-\beta_{2} y+\rho \frac{t^{\alpha}}{\alpha}+\theta\right\}} .
\end{aligned}
$$

Case II:

$$
A_{0}= \pm A_{1} \sqrt{\frac{\rho+\mu \beta_{1}^{2}+\eta \beta_{1} \beta_{2}+\lambda \beta_{2}^{2}}{\delta}}, \quad A_{2}=0, \quad \nu= \pm \sqrt{\frac{\rho+\mu \beta_{1}^{2}+\eta \beta_{1} \beta_{2}+\lambda \beta_{2}^{2}}{2\left(\mu \alpha_{1}^{2}+\eta \alpha_{1} \alpha_{2}+\lambda \alpha_{2}^{2}\right)}} .
$$

Again, substituting these results into Eq. (5) by using Eq. (8), we obtain

$$
\begin{gathered}
\Phi_{2,5}(x, y, t)=\sqrt{\frac{\rho+\mu \beta_{1}^{2}+\eta \beta_{1} \beta_{2}+\lambda \beta_{2}^{2}}{\delta}}\left(\cot \left[\sqrt{\frac{\rho+\mu \beta_{1}^{2}+\eta \beta_{1} \beta_{2}+\lambda \beta_{2}^{2}}{2\left(\mu \alpha_{1}^{2}+\eta \alpha_{1} \alpha_{2}+\lambda \alpha_{2}^{2}\right)}}(s)\right]\right) e^{i\left\{-\beta_{1} x-\beta_{2} y+\rho \frac{t^{\alpha}}{\alpha}+\theta\right\}} . \\
\Phi_{2,6}(x, y, t)=-\sqrt{\frac{\rho+\mu \beta_{1}^{2}+\eta \beta_{1} \beta_{2}+\lambda \beta_{2}^{2}}{\delta}}\left(\cot \left[\sqrt{\frac{\rho+\mu \beta_{1}^{2}+\eta \beta_{1} \beta_{2}+\lambda \beta_{2}^{2}}{2\left(\mu \alpha_{1}^{2}+\eta \alpha_{1} \alpha_{2}+\lambda \alpha_{2}^{2}\right)}}(s)\right]\right) e^{i\left\{-\beta_{1} x-\beta_{2} y+\rho \frac{t^{\alpha}}{\alpha}+\theta\right\}},
\end{gathered}
$$

provided that $\left(\rho+\mu \beta_{1}^{2}+\eta \beta_{1} \beta_{2}+\lambda \beta_{2}^{2}\right)\left(\mu \alpha_{1}^{2}+\eta \alpha_{1} \alpha_{2}+\lambda \alpha_{2}^{2}\right)>0$.

\subsection{Applications to the extended rational sinh-cosh method}

Assume that Eq. (6) has the solution of the form:

$$
\Psi(s)=\frac{A_{0} \sinh (\nu s)}{A_{2}+A_{1} \cosh (\nu s)}, \quad \cosh (\nu s) \neq-\frac{A_{2}}{A_{1}},
$$

or of the form

$$
\Psi(s)=\frac{A_{0} \cosh (\nu s)}{A_{2}+A_{1} \sinh (\nu s)}, \quad \sinh (\nu s) \neq-\frac{A_{2}}{A_{1}},
$$

where $A_{0}, A_{1}$ and $A_{2}$ are parameters that will be determined and $\nu$ represents wave number.

Family I

Now, substituting Eq. (21) into Eq. (6) and then setting each coefficients of all terms of $\cosh ^{m}(\nu s)$ or $\sinh ^{m}(\nu s)$ to zero, yields a system of algebraic equations. Then we obtain system of algebraic equations involving parameters $A_{0}, A_{1}, A_{2}, \mu, \nu$, $\lambda, \delta, \rho$. This system of equations are solved as follows:

$$
\begin{aligned}
\delta A_{0}^{2}+A_{1}^{2}\left(\rho+\mu \beta_{1}^{2}+\eta \beta_{1} \beta_{2}+\lambda \beta_{2}^{2}\right) & =0, \\
A_{1} A_{2}\left[\mu \nu^{2} \alpha_{1}^{2}+\eta \nu^{2} \alpha_{1} \alpha_{2}+\lambda \nu^{2} \alpha_{2}^{2}+2\left(\rho+\mu \beta_{1}^{2}+\eta \beta_{1} \beta_{2}+\lambda \beta_{2}^{2}\right)\right] & =0, \\
-\delta A_{0}^{2}+2 \nu^{2} A_{1}^{2}\left(\mu \alpha_{1}^{2}+\eta \alpha_{1} \alpha_{2}+\lambda \alpha_{2}^{2}\right)+A_{2}^{2}\left(\rho-\mu \nu^{2} \alpha_{1}^{2}-\eta \nu^{2} \alpha_{1} \alpha_{2}-\lambda \nu^{2} \alpha_{2}^{2}+\mu \beta_{1}^{2}+\eta \beta_{1} \beta_{2}+\lambda \beta_{2}^{2}\right) & =0 .
\end{aligned}
$$


Solving this system, we yields the following set of solutions with help of Mathematica: Case I:

$$
A_{0}= \pm i A_{1} \sqrt{\frac{\rho+\mu \beta_{1}^{2}+\eta \beta_{1} \beta_{2}+\lambda \beta_{2}^{2}}{\delta}}, \quad A_{1}= \pm A_{2}, \quad \nu= \pm \sqrt{\frac{-2\left(\rho+\mu \beta_{1}^{2}+\eta \beta_{1} \beta_{2}+\lambda \beta_{2}^{2}\right)}{\mu \alpha_{1}^{2}+\eta \alpha_{1} \alpha_{2}+\lambda \alpha_{2}^{2}}} .
$$

Substituting these results into Eq. (5) by using Eq. (21), we have

$$
\begin{aligned}
\Phi_{3,1}(x, y, t)= & i \sqrt{\frac{\rho+\mu \beta_{1}^{2}+\eta \beta_{1} \beta_{2}+\lambda \beta_{2}^{2}}{\delta}}\left(\frac{\sinh \left[\sqrt{\frac{-2\left(\rho+\mu \beta_{1}^{2}+\eta \beta_{1} \beta_{2}+\lambda \beta_{2}^{2}\right)}{\mu \alpha_{1}^{2}+\eta \alpha_{1} \alpha_{2}+\lambda \alpha_{2}^{2}}}(s)\right]}{1+\cosh \left[\sqrt{\frac{-2\left(\rho+\mu \beta_{1}^{2}+\eta \beta_{1} \beta_{2}+\lambda \beta_{2}^{2}\right)}{\mu \alpha_{1}^{2}+\eta \alpha_{1} \alpha_{2}+\lambda \alpha_{2}^{2}}}(s)\right]}\right] \\
& \times e^{i\left\{-\beta_{1} x-\beta_{2} y+\rho \frac{t^{\alpha}}{\alpha}+\theta\right\} .} \\
\Phi_{3,2}(x, y, t) & =-i \sqrt{\frac{\rho+\mu \beta_{1}^{2}+\eta \beta_{1} \beta_{2}+\lambda \beta_{2}^{2}}{\delta}}\left(\frac{\sinh \left[\sqrt{\frac{-2\left(\rho+\mu \beta_{1}^{2}+\eta \beta_{1} \beta_{2}+\lambda \beta_{2}^{2}\right)}{\mu \alpha_{1}^{2}+\eta \alpha_{1} \alpha_{2}+\lambda \alpha_{2}^{2}}}(s)\right]}{1+\cosh \left[\sqrt{\frac{-2\left(\rho+\mu \beta_{1}^{2}+\eta \beta_{1} \beta_{2}+\lambda \beta_{2}^{2}\right)}{\mu \alpha_{1}^{2}+\eta \alpha_{1} \alpha_{2}+\lambda \alpha_{2}^{2}}}(s)\right]}\right) \\
& \times e^{i\left\{-\beta_{1} x-\beta_{2} y+\rho \frac{t^{\alpha}}{\alpha}+\theta\right\} .} \\
\Phi_{3,3}(x, y, t) & =i \sqrt{\frac{\rho+\mu \beta_{1}^{2}+\eta \beta_{1} \beta_{2}+\lambda \beta_{2}^{2}}{\delta}}\left(\frac{\sinh \left[\sqrt{\frac{-2\left(\rho+\mu \beta_{1}^{2}+\eta \beta_{1} \beta_{2}+\lambda \beta_{2}^{2}\right)}{\mu \alpha_{1}^{2}+\eta \alpha_{1} \alpha_{2}+\lambda \alpha_{2}^{2}}}(s)\right]}{1-\cosh \left[\sqrt{\frac{-2\left(\rho+\mu \beta_{1}^{2}+\eta \beta_{1} \beta_{2}+\lambda \beta_{2}^{2}\right)}{\mu \alpha_{1}^{2}+\eta \alpha_{1} \alpha_{2}+\lambda \alpha_{2}^{2}}}(s)\right]}\right) \\
& \times e^{i\left\{-\beta_{1} x-\beta_{2} y+\rho \frac{t^{\alpha}}{\alpha}+\theta\right\} .} \\
\Phi_{3,4}(x, y, t) & =-i \sqrt{\frac{\rho+\mu \beta_{1}^{2}+\eta \beta_{1} \beta_{2}+\lambda \beta_{2}^{2}}{\delta}}\left(\frac{\sinh \left[\sqrt{\frac{-2\left(\rho+\mu \beta_{1}^{2}+\eta \beta_{1} \beta_{2}+\lambda \beta_{2}^{2}\right)}{\mu \alpha_{1}^{2}+\eta \alpha_{1} \alpha_{2}+\lambda \alpha_{2}^{2}}}(s)\right]}{1-\cosh \left[\sqrt{\frac{-2\left(\rho+\mu \beta_{1}^{2}+\eta \beta_{1} \beta_{2}+\lambda \beta_{2}^{2}\right)}{\mu \alpha_{1}^{2}+\eta \alpha_{1} \alpha_{2}+\lambda \alpha_{2}^{2}}}(s)\right]}\right)
\end{aligned}
$$

Case II:

$$
A_{0}= \pm i A_{1} \sqrt{\frac{\rho+\mu \beta_{1}^{2}+\eta \beta_{1} \beta_{2}+\lambda \beta_{2}^{2}}{\delta}}, \quad A_{2}=0, \quad \nu= \pm \sqrt{\frac{-\left(\rho+\mu \beta_{1}^{2}+\eta \beta_{1} \beta_{2}+\lambda \beta_{2}^{2}\right)}{2\left(\mu \alpha_{1}^{2}+\eta \alpha_{1} \alpha_{2}+\lambda \alpha_{2}^{2}\right)}}
$$

Again, substituting these results for only the positive values into Eq. (21), we obtain

$$
\begin{aligned}
& \Phi_{3,5}(x, y, t)=i \sqrt{\frac{\rho+\mu \beta_{1}^{2}+\eta \beta_{1} \beta_{2}+\lambda \beta_{2}^{2}}{\delta}}\left(\tanh \left[\sqrt{\frac{-\left(\rho+\mu \beta_{1}^{2}+\eta \beta_{1} \beta_{2}+\lambda \beta_{2}^{2}\right)}{2\left(\mu \alpha_{1}^{2}+\eta \alpha_{1} \alpha_{2}+\lambda \alpha_{2}^{2}\right)}}(s)\right]\right) e^{i\left\{-\beta_{1} x-\beta_{2} y+\rho \frac{t^{\alpha}}{\alpha}+\theta\right\}} . \\
& \Phi_{3,6}(x, y, t)=-i \sqrt{\frac{\rho+\mu \beta_{1}^{2}+\eta \beta_{1} \beta_{2}+\lambda \beta_{2}^{2}}{\delta}}\left(\tanh \left[\sqrt{\frac{-\left(\rho+\mu \beta_{1}^{2}+\eta \beta_{1} \beta_{2}+\lambda \beta_{2}^{2}\right)}{2\left(\mu \alpha_{1}^{2}+\eta \alpha_{1} \alpha_{2}+\lambda \alpha_{2}^{2}\right)}}(s)\right]\right) e^{i\left\{-\beta_{1} x-\beta_{2} y+\rho \frac{t^{\alpha}}{\alpha}+\theta\right\}},
\end{aligned}
$$

holds for $\left(\rho+\mu \beta_{1}^{2}+\eta \beta_{1} \beta_{2}+\lambda \beta_{2}^{2}\right)\left(\mu \alpha_{1}^{2}+\eta \alpha_{1} \alpha_{2}+\lambda \alpha_{2}^{2}\right)<0$. 
Family II

Again, substituting Eq. (22) into Eq. (6) and then setting each coefficients of all terms of $\sinh ^{m}(\nu s) \operatorname{or~} \cosh ^{m}(\nu s)$ to zero, yields a system of algebraic equations. Then we obtain system of algebraic equations involving parameters $A_{0}, A_{1}, A_{2}, \mu, \nu$, $\lambda, \delta, \rho$.

$$
\begin{aligned}
\delta A_{0}^{2}+A_{1}^{2}\left(\rho+\mu \beta_{1}^{2}+\eta \beta_{1} \beta_{2}+\lambda \beta_{2}^{2}\right) & =0, \\
A_{1} A_{2}\left[\mu \nu^{2} \alpha_{1}^{2}+\eta \nu^{2} \alpha_{1} \alpha_{2}+\lambda \nu^{2} \alpha_{2}^{2}+2\left(\rho+\mu \beta_{1}^{2}+\eta \beta_{1} \beta_{2}+\lambda \beta_{2}^{2}\right)\right] & =0, \\
\delta A_{0}^{2}-2 \nu^{2} A_{1}^{2}\left(\mu \alpha_{1}^{2}+\eta \alpha_{1} \alpha_{2}+\lambda \alpha_{2}^{2}\right)+A_{2}^{2}\left(\rho-\mu \nu^{2} \alpha_{1}^{2}-\eta \nu^{2} \alpha_{1} \alpha_{2}-\lambda \nu^{2} \alpha_{2}^{2}+\mu \beta_{1}^{2}+\eta \beta_{1} \beta_{2}+\lambda \beta_{2}^{2}\right) & =0 .
\end{aligned}
$$

This system, gives the following set of solutions:

Case I:

$$
A_{0}= \pm i A_{1} \sqrt{\frac{\rho+\mu \beta_{1}^{2}+\eta \beta_{1} \beta_{2}+\lambda \beta_{2}^{2}}{\delta}}, \quad A_{1}= \pm A_{2}, \quad \nu= \pm \sqrt{\frac{-2\left(\rho+\mu \beta_{1}^{2}+\eta \beta_{1} \beta_{2}+\lambda \beta_{2}^{2}\right)}{\mu \alpha_{1}^{2}+\eta \alpha_{1} \alpha_{2}+\lambda \alpha_{2}^{2}}} .
$$

Substituting these results into Eq. (5) by using Eq. (22), we obtain

$$
\begin{aligned}
\Phi_{4,1}(x, y, t) & =i \sqrt{\frac{\rho+\mu \beta_{1}^{2}+\eta \beta_{1} \beta_{2}+\lambda \beta_{2}^{2}}{\delta}}\left(\frac{\cosh \left[\sqrt{\frac{-2\left(\rho+\mu \beta_{1}^{2}+\eta \beta_{1} \beta_{2}+\lambda \beta_{2}^{2}\right)}{\mu \alpha_{1}^{2}+\eta \alpha_{1} \alpha_{2}+\lambda \alpha_{2}^{2}}}(s)\right]}{1+\sinh \left[\sqrt{\frac{-2\left(\rho+\mu \beta_{1}^{2}+\eta \beta_{1} \beta_{2}+\lambda \beta_{2}^{2}\right)}{\mu \alpha_{1}^{2}+\eta \alpha_{1} \alpha_{2}+\lambda \alpha_{2}^{2}}}(s)\right]}\right) \\
& \times e^{i\left\{-\beta_{1} x-\beta_{2} y+\rho \frac{t^{\alpha}}{\alpha}+\theta\right\}} .
\end{aligned}
$$$$
\Phi_{4,2}(x, y, t)=-i \sqrt{\frac{\rho+\mu \beta_{1}^{2}+\eta \beta_{1} \beta_{2}+\lambda \beta_{2}^{2}}{\delta}}\left(\frac{\cosh \left[\sqrt{\frac{-2\left(\rho+\mu \beta_{1}^{2}+\eta \beta_{1} \beta_{2}+\lambda \beta_{2}^{2}\right)}{\mu \alpha_{1}^{2}+\eta \alpha_{1} \alpha_{2}+\lambda \alpha_{2}^{2}}}(s)\right]}{1+\sinh \left[\sqrt{\frac{-2\left(\rho+\mu \beta_{1}^{2}+\eta \beta_{1} \beta_{2}+\lambda \beta_{2}^{2}\right)}{\mu \alpha_{1}^{2}+\eta \alpha_{1} \alpha_{2}+\lambda \alpha_{2}^{2}}}(s)\right]}\right)
$$$$
\times e^{i\left\{-\beta_{1} x-\beta_{2} y+\rho \frac{t^{\alpha}}{\alpha}+\theta\right\}} .
$$

$$
\begin{aligned}
\Phi_{4,3}(x, y, t) & =i \sqrt{\frac{\rho+\mu \beta_{1}^{2}+\eta \beta_{1} \beta_{2}+\lambda \beta_{2}^{2}}{\delta}}\left(\frac{\cosh \left[\sqrt{\frac{-2\left(\rho+\mu \beta_{1}^{2}+\eta \beta_{1} \beta_{2}+\lambda \beta_{2}^{2}\right)}{\mu \alpha_{1}^{2}+\eta \alpha_{1} \alpha_{2}+\lambda \alpha_{2}^{2}}}(s)\right]}{1-\sinh \left[\sqrt{\frac{-2\left(\rho+\mu \beta_{1}^{2}+\eta \beta_{1} \beta_{2}+\lambda \beta_{2}^{2}\right)}{\mu \alpha_{1}^{2}+\eta \alpha_{1} \alpha_{2}+\lambda \alpha_{2}^{2}}}(s)\right]}\right) \\
& \times e^{i\left\{-\beta_{1} x-\beta_{2} y+\rho \frac{t^{\alpha}}{\alpha}+\theta\right\}} .
\end{aligned}
$$

$$
\begin{aligned}
\Phi_{4,4}(x, y, t) & =-i \sqrt{\frac{\rho+\mu \beta_{1}^{2}+\eta \beta_{1} \beta_{2}+\lambda \beta_{2}^{2}}{\delta}}\left(\frac{\cosh \left[\sqrt{\frac{-2\left(\rho+\mu \beta_{1}^{2}+\eta \beta_{1} \beta_{2}+\lambda \beta_{2}^{2}\right)}{\mu \alpha_{1}^{2}+\eta \alpha_{1} \alpha_{2}+\lambda \alpha_{2}^{2}}}(s)\right]}{1-\sinh \left[\sqrt{\frac{-2\left(\rho+\mu \beta_{1}^{2}+\eta \beta_{1} \beta_{2}+\lambda \beta_{2}^{2}\right)}{\mu \alpha_{1}^{2}+\eta \alpha_{1} \alpha_{2}+\lambda \alpha_{2}^{2}}}(s)\right]}\right) \\
& \times e^{i\left\{-\beta_{1} x-\beta_{2} y+\rho \frac{t^{\alpha}}{\alpha}+\theta\right\}} .
\end{aligned}
$$

Case-II:

$$
A_{0}= \pm i A_{1} \sqrt{\frac{\rho+\mu \beta_{1}^{2}+\eta \beta_{1} \beta_{2}+\lambda \beta_{2}^{2}}{\delta}}, \quad A_{2}=0, \quad \nu= \pm \sqrt{\frac{-\left(\rho+\mu \beta_{1}^{2}+\eta \beta_{1} \beta_{2}+\lambda \beta_{2}^{2}\right)}{2\left(\mu \alpha_{1}^{2}+\eta \alpha_{1} \alpha_{2}+\lambda \alpha_{2}^{2}\right)}} .
$$


Again, substituting these results into Eq. (5) by using Eq. (22), we obtain

$$
\begin{aligned}
& \Phi_{4,5}(x, y, t)=i \sqrt{\frac{\rho+\mu \beta_{1}^{2}+\eta \beta_{1} \beta_{2}+\lambda \beta_{2}^{2}}{\delta}}\left(\operatorname{coth}\left[\sqrt{\frac{-\left(\rho+\mu \beta_{1}^{2}+\eta \beta_{1} \beta_{2}+\lambda \beta_{2}^{2}\right)}{2\left(\mu \alpha_{1}^{2}+\eta \alpha_{1} \alpha_{2}+\lambda \alpha_{2}^{2}\right)}}(s)\right]\right) e^{i\left\{-\beta_{1} x-\beta_{2} y+\rho \frac{t^{\alpha}}{\alpha}+\theta\right\}} . \\
& \Phi_{4,5}(x, y, t)=-i \sqrt{\frac{\rho+\mu \beta_{1}^{2}+\eta \beta_{1} \beta_{2}+\lambda \beta^{2}}{\delta}}\left(\operatorname{coth}\left[\sqrt{\frac{-\left(\rho+\mu \beta_{1}^{2}+\eta \beta_{1} \beta_{2}+\lambda \beta_{2}^{2}\right)}{2\left(\mu \alpha_{1}^{2}+\eta \alpha_{1} \alpha_{2}+\lambda \alpha_{2}^{2}\right)}}(s)\right]\right) e^{i\left\{-\beta_{1} x-\beta_{2} y+\rho \frac{t^{\alpha}}{\alpha}+\theta\right\}}
\end{aligned}
$$

provided that $\left(\rho+\mu \beta_{1}^{2}+\eta \beta_{1} \beta_{2}+\lambda \beta_{2}^{2}\right)\left(\mu \alpha_{1}^{2}+\eta \alpha_{1} \alpha_{2}+\lambda \alpha_{2}^{2}\right)>0$.

\section{Physical significance and graphical representations}

In this section, we construct the physical interpretation to the some problem of the paper that add extra flavour to our analytical solutions of (2). For this purpose, graphical representations of some established problem are discussed and suitable choice of help us to construct dark, singular and traveling wave solutions. The graphically representations of some obtained solutions in two and three-dimensional are given from Figs. 1 to 7. Figure 1 for Eq. (13) and Fig. (2) for Eq. (19) represent periodic wave solutions and two-dimensional graphics limit cycle with suitable choice of parameters. Figure 5 for Eq. (27) and Fig. 7 for Eq. (33) show dark and singular wave solutions. Dark and singular wave solutions types presents in Figs. 3, 4 for Eq. (23) and Fig. 6 for Eq. (29).
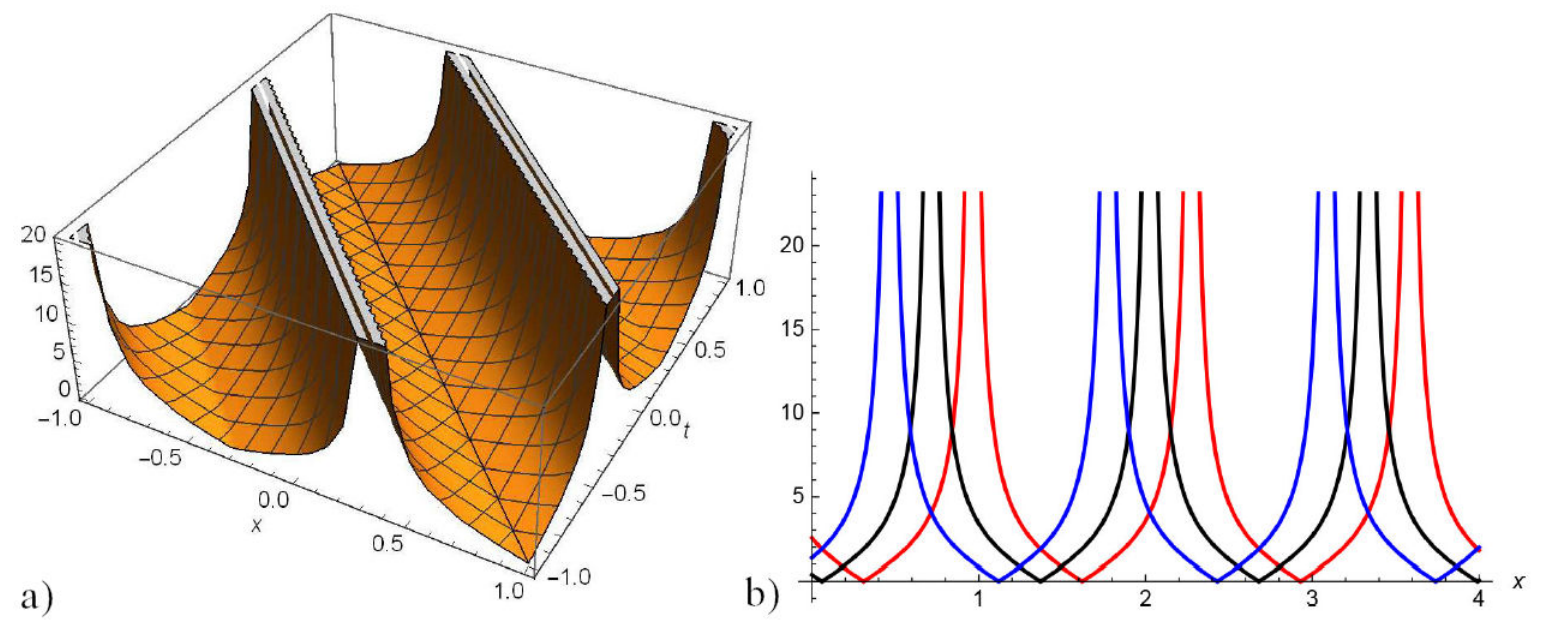

FIGURE 1. a) $3 \mathrm{D}$ and b) $2 \mathrm{D}$ surfaces for the $\left|\Phi_{1,5}(x, y, t)\right|$ with $-1 \leq x, t \leq 1$ for the values $\mu=1, \eta=2.50, \lambda=2.50, \delta=1.50, \alpha=1$, $\alpha_{1}=-1, \alpha_{2}=1, \beta_{1}=2, \beta_{2}=1, \theta=0, \rho=0, \omega=1, y=0$ and their projections at $t=1,1.25,1.50$.

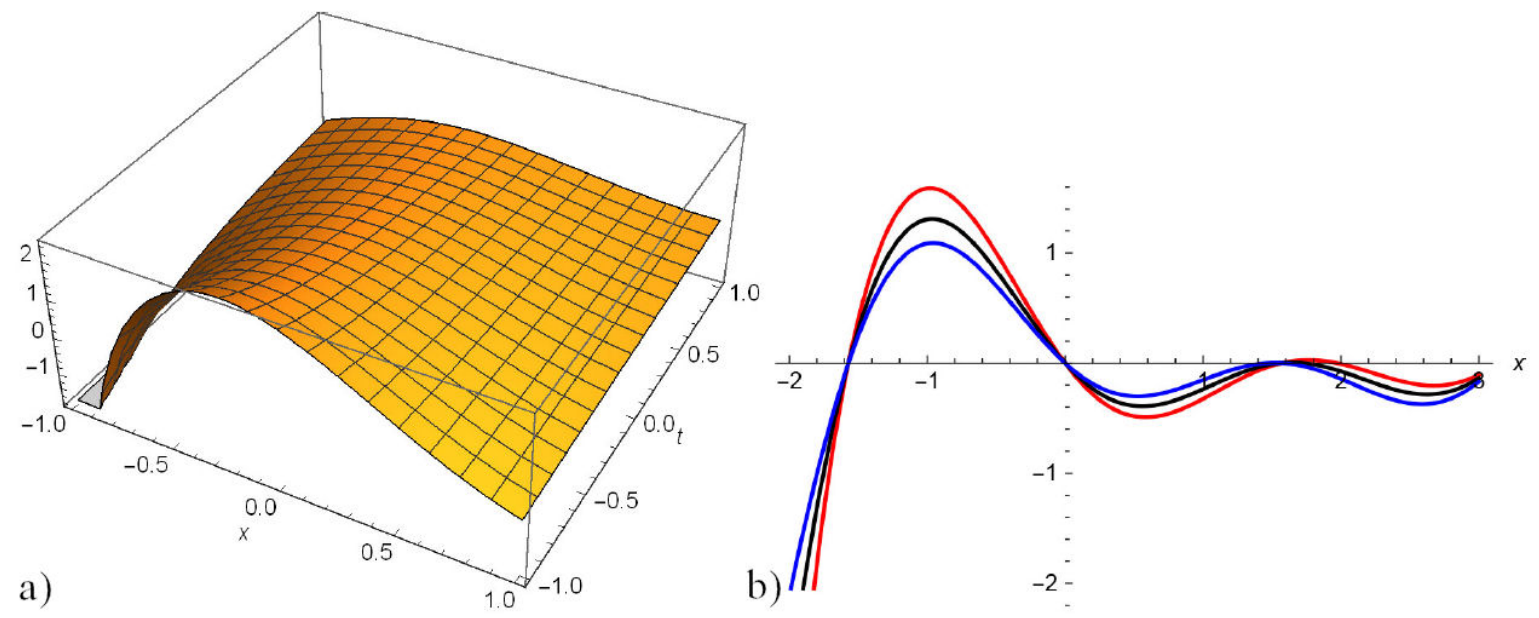

FIGURE 2. a) 3D and b) 2D surfaces of $\left|\Phi_{2,5}(x, y, t)\right|$ with $-1 \leq x, t \leq 1$ for the values $\mu=1, \eta=2.50, \lambda=2.50, \delta=1.50, \alpha=1$, $\alpha_{1}=1, \alpha_{2}=1, \beta_{1}=2, \beta_{2}=-1, \theta=0 \rho=0, \omega=-1, y=0$ and their projections at $t=1,2,3$. 


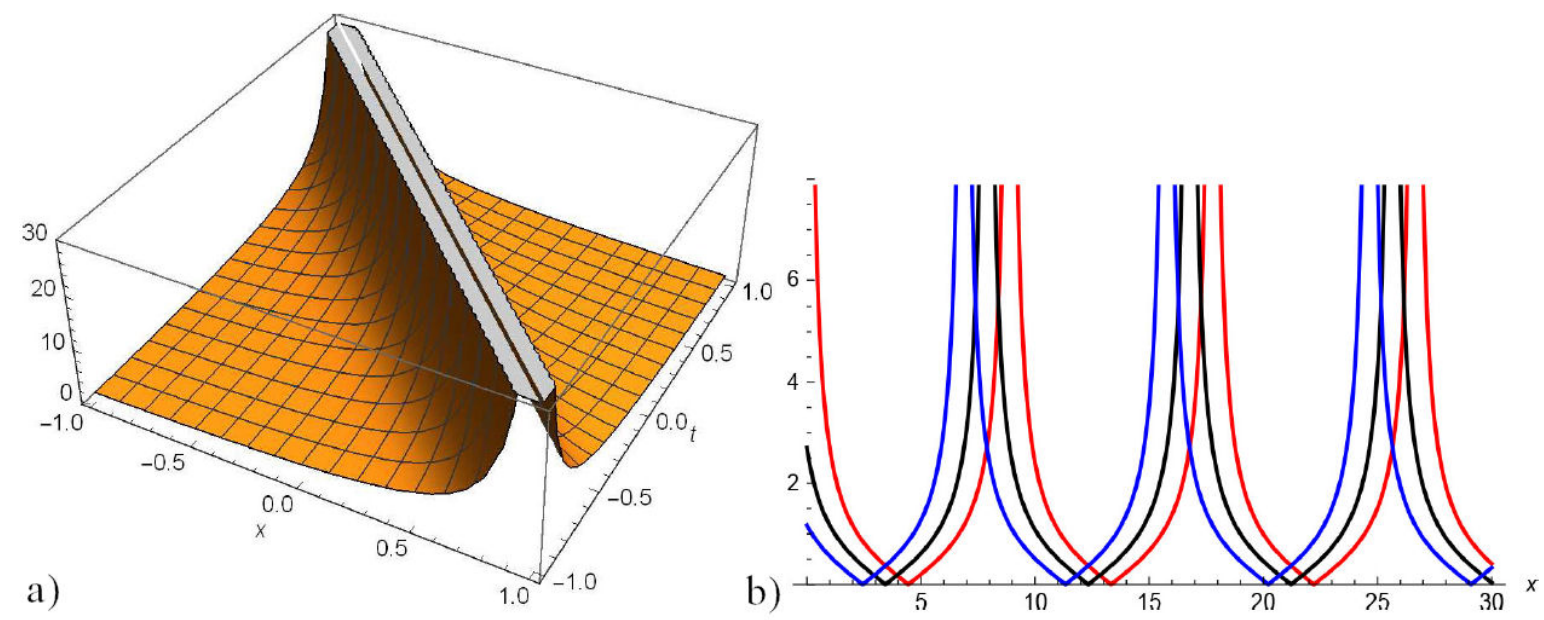

FIGURE 3. a) 3D and b) 2D surfaces of imaginary part of the $\Phi_{3,1}(x, y, t)$ with $-1 \leq x, t \leq 1$ for the values $\mu=1, \eta=2.50, \lambda=2.50$, $\delta=-1.50, \alpha=1, \alpha_{1}=1.70, \alpha_{2}=1, \beta_{1}=2, \beta_{2}=-1, \theta=0 \rho=-4, \omega=1, y=2$ and their projections at $t=0.25,0.500 .75$.

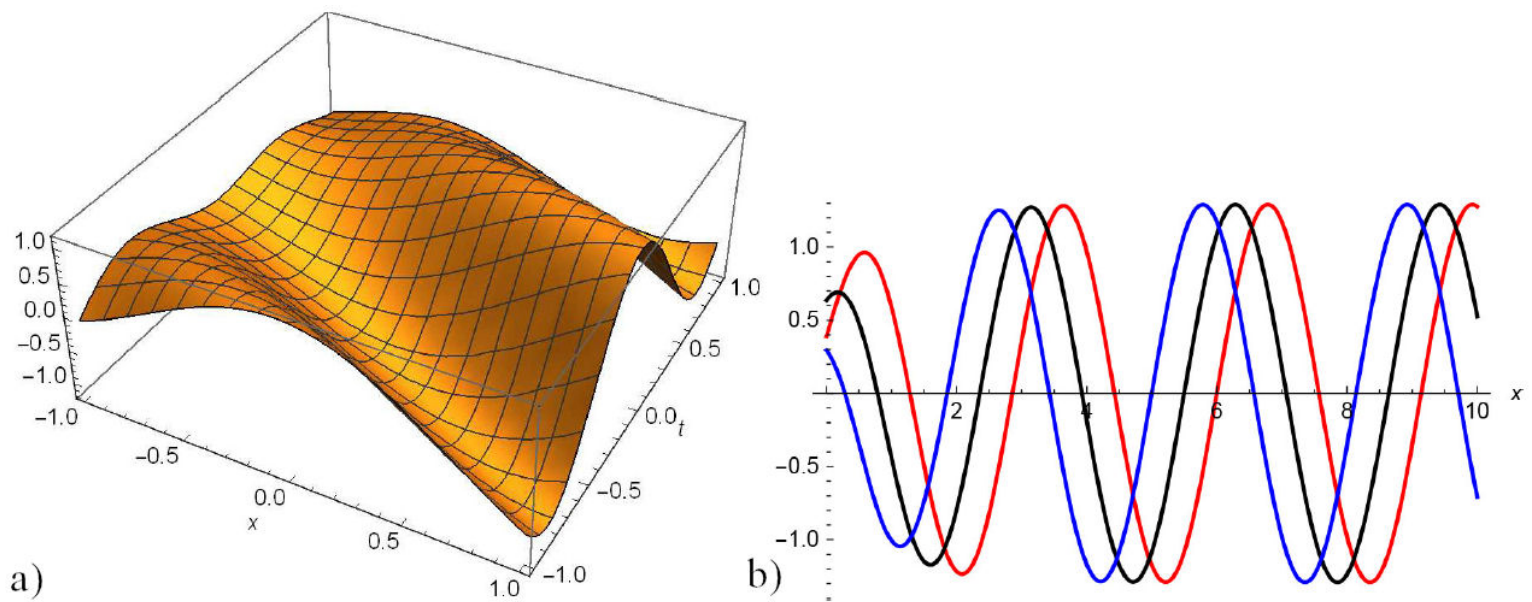

FIGURE 4. a) 3D and b) 2D surfaces of real part of the $\Phi_{3,1}(x, y, t)$ with $-5 \leq x, t \leq 5$ for the values $\mu=1, \eta=2.50, \lambda=2.50$, $\delta=-1.50, \alpha=1, \alpha_{1}=1.70, \alpha_{2}=1, \beta_{1}=2, \beta_{2}=-1.70, \theta=0 \rho=-4, \omega=1, y=-2$ and their projections at $t=0.25,0.50,0.75$.

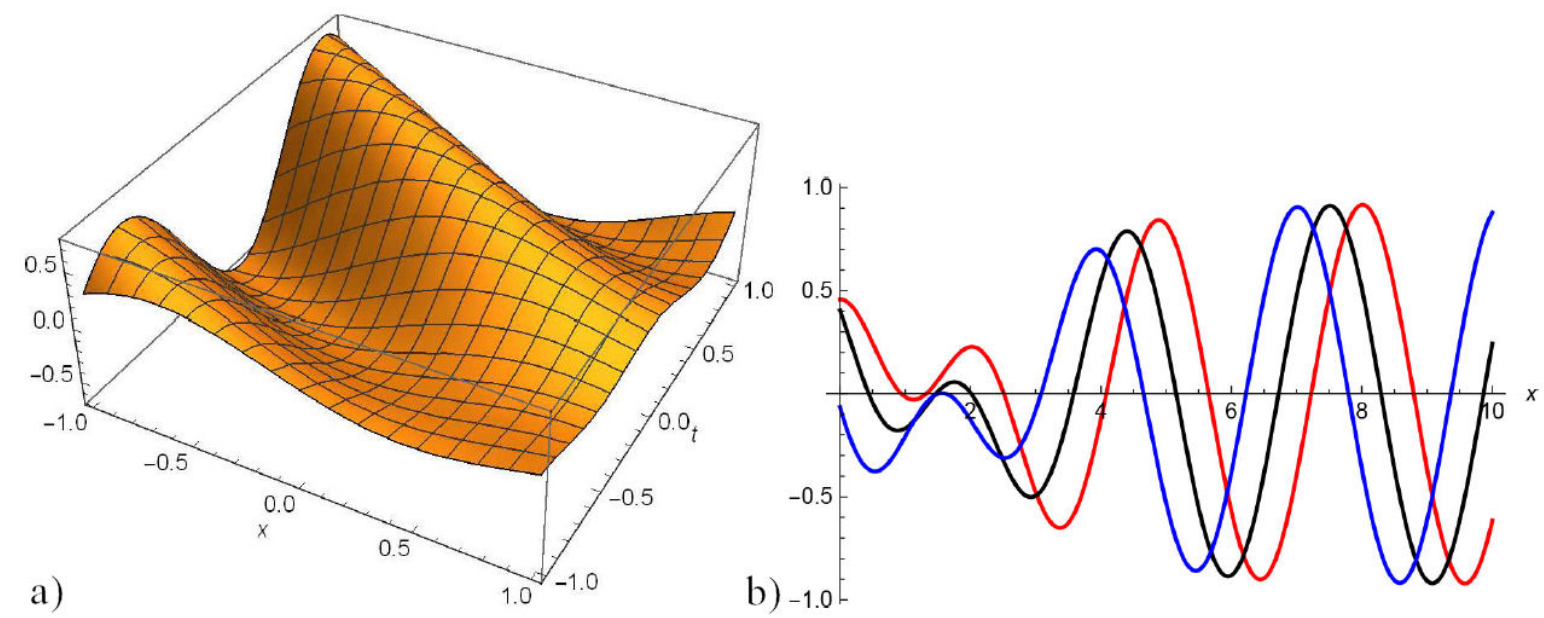

FIGURE 5. a) 3D and b) 2D surfaces of $\left|\Phi_{3,5}(x, y, t)\right|$ with $-1 \leq x, t \leq 1$ for the values $\mu=1, \eta=2.50, \lambda=2.50, \delta=-1.50, \alpha=1$, $\alpha_{1}=-1, \alpha_{2}=1, \beta_{1}=2, \beta_{2}=-1, \rho=-2, \omega=1, y=0$ and their projections at $t=0,2,4$. 


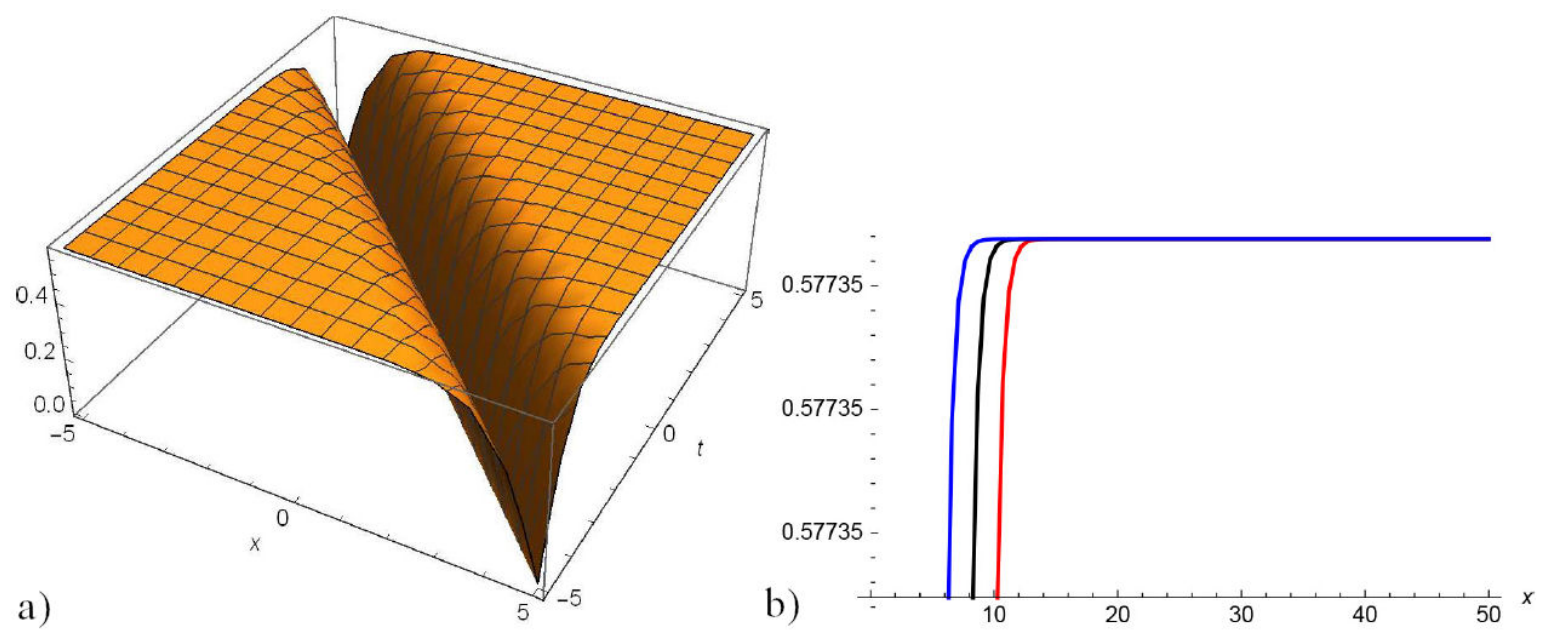

FIGURE 6. a) 3D and b) 2D surfaces of $\left|\Phi_{4,1}(x, y, t)\right|$ with $-3 \leq x, t \leq 3$ for the values $\mu=1, \eta=2.50, \lambda=2.50, \delta=1.50, \alpha=1$, $\alpha_{1}=1.70, \alpha_{2}=1, \beta_{1}=-1, \beta_{2}=1, \theta=0, \rho=-4, \omega=1, y=1$ and their projections at $t=1,2,3$.

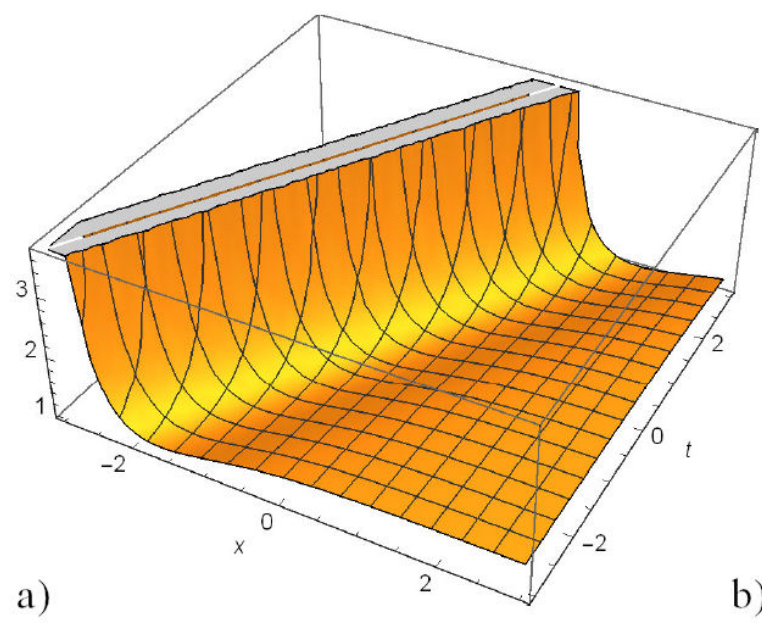

b)

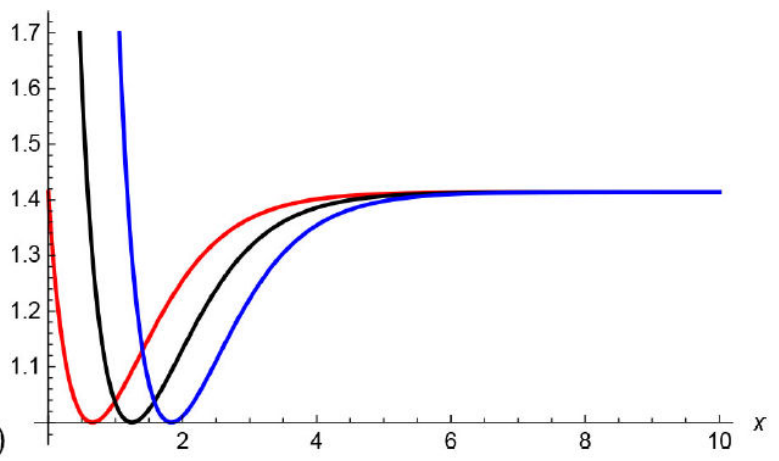

FIGURE 7. a) 3D and b) 2D surfaces of $\left|\Phi_{4,5}(x, y, t)\right|$ with $-3 \leq x, t \leq 3$ for the values $\mu=1, \eta=2.50, \lambda=0, \delta=1.50, \alpha=1$, $\alpha_{1}=1.70, \alpha_{2}=1, \beta_{1}=-1, \beta_{2}=1, \theta=0 \rho=-4, \omega=-1, y=0$ and their projections at $t=0,1,2$.

\section{Concluding remarks}

In our work, we have employed suitable technique to develop some new travelling wave solutions to the special kind of nonlinear Schrödinger's equations. The extended rational sine-cosine method and extended rational sinh-cosh method are found to be as one of the most effective, accurate and powerful tools for the construction of analytical solutions for fractional HFSCs in the semi-classical limit. As a result, obtained some new exact solution in the form of hyperbolic, trigonometric and complex functions solutions. On the basis of our results, we found that solutions presented in [37-39] using different models these solutions will be useful in future development in order to construct exact solutions, the existence criteria of involving parameters are also discussed. Moreover, some of exact solutions obtained by these methods are mostly identical. We can conclude that the proposed method is one of the most proficient techniques and can be efficiently employed for further investigation to NPDEs rasing in contemporary science.
1. M. M. Khater, R. A. Attia, C. Park, D. Lu, On the numerical investigation of the interaction in plasma between (high and low) frequency of (langmuir and ion-acoustic) waves, Results in Physics 18 (2020) 103317.
2. M. M. Khater, R. A. Attia, D. Lu, Computational and numerical simulations for the nonlinear fractional KolmogorovPetrovskii-Piskunov (FKPP) equation, Physica Scripta 95 (2020) 055213. 
3. B. Romanowicz, L.-W. Chen, S. W. French, Accelerating full waveform inversion via source stacking and cross-correlations, Geophysical Journal International 220 (2020) 308.

4. H. Günerhan, F. S. Khodadad, H. Rezazadeh, M. M. Khater, Exact optical solutions of the $(2+1)$ dimensions kundumukherjee-naskar model via the new extended direct algebraic method, Modern Physics Letters B 34 (2020) 2050225.

5. M. Hassan, R. Ellahi, A. Zeeshan, M. M. Bhatti, Analysis of natural convective ow of non-newtonian uid under the effects of nanoparticles of different materials, Proceedings of the Institution of Mechanical Engineers, Part E: Journal of Process Mechanical Engineering 233 (2019) 643.

6. S. Chen, Y. Zhou, L. Bu, F. Baronio, J. M. Soto-Crespo, D. Mihalache, Super chirped rogue waves in optical fibers, Optics express 27 (2019) 11370.

7. H. U. Rehman, M. Younis, S. Jafar, M. Tahir, M. S. Saleem, Optical solitons of biswas-arshed model in birefrigent fiber without four wave mixing, Optik (2020) 164669.

8. M. M. Khater, R. A. Attia, D. Baleanu, Abundant new solutions of the transmission of nerve impulses of an excitable system, The European Physical Journal Plus 135 (2020) 1.

9. Y. Wang, P. Verma, L. Zhang, Y. Li, Z. Liu, D. G. Truhlar, X. He, M06-sx screened-exchange density functional for chemistry and solid-state physics, Proceedings of the National Academy of Sciences (2020).

10. T.-T. Jia, Y.-T. Gao, Y.-J. Feng, L. Hu, J.-J. Su, L.-Q. Li, C.C. Ding, On the quintic time-dependent coefficient derivative nonlinear Schrödinger equation in hydrodynamics or fiber optics, Nonlinear Dynamics 96 (2019) 229.

11. A.-H. Abdel-Aty, M. M. Khater, D. Baleanu, E. Khalil, J. Bouslimi, M. Omri, Abundant distinct types of solutions for the nervous biological fractional Fitzhugh-Nagumo equation via three different sorts of schemes, Advances in Difference Equations 2020 (2020) 1.

12. M. M. Khater, R. A. Attia, S. S. Alodhaibi, D. Lu, Novel soliton waves of two uid nonlinear evolutions models in the view of computational scheme, International Journal of Modern Physics B 34 (2020) 2050096.

13. L. Qian, R. A. Attia, Y. Qiu, D. Lu, M. M. Khater, The shock peakon wave solutions of the general Degasperis-Procesi equation, International Journal of Modern Physics B 33 (2019) 1950351.

14. A. Amiri, A. Cordero, M. T. Darvishi, J. R. Torregrosa, A fast algorithm to solve systems of nonlinear equations, Journal of Computational and Applied Mathematics 354 (2019) 242.

15. X. Zhang, Y. Chen, Inverse scattering transformation for generalized nonlinear Schrödinger equation, Applied Mathematics Letters 98 (2019) 306.

16. X.-X. Li, C.-H. He, Homotopy perturbation method coupled with the enhanced perturbation method, Journal of Low Frequency Noise, Vibration and Active Control 38 (2019) 1399.

17. S. B.Munusamy, A. Dhar, On use of expanding parameters and auxiliary term in homotopy perturbation method for boussinesq equation with tidal condition, Environmental Modeling and Assessment 24 (2019) 109.
18. X. Guan, W. Liu, Q. Zhou, A. Biswas, Darboux transformation and analytic solutions for a generalized super-nls-mkdv equation, Nonlinear Dynamics 98 (2019) 1491.

19. X. Xin, Y. Xia, H. Liu, L. Zhang, Darboux transformation of the variable coefficient nonlocal equation, Journal of Mathematical Analysis and Applications (2020) 124227.

20. C. Yue, M. M. Khater, R. A. Attia, D. Lu, Computational simulations of the couple Boiti-Leon-Pempinelli (BLP) system and the (3+1)-dimensional Kadomtsev-Petviashvili (KP) equation, AIP Advances 10 (2020) 045216.

21. C. Yue, M. M. Khater, M. Inc, R. A. Attia, D. Lu, Abundant analytical solutions of the fractional nonlinear $(2+1)$ dimensional blmp equation arising in incompressible uid, International Journal of Modern Physics B 34 (2020) 2050084.

22. M. M. Khater, C. Park, D. Lu, Two effective computational schemes for a prototype of an excitable system, AIP Advances 10 (2020) 105120.

23. M. M. Khater, D.-C. Lu, R. A. Attia, M. Inç, Analytical and approximate solutions for complex nonlinear Schrödinger equation via generalized auxiliary equation and numerical schemes, Communications in Theoretical Physics 71 (2019) 1267.

24. T. A. Sulaiman, H. Bulut, The new extended rational sgeem for construction of optical solitons to the $(2+1)$-dimensional kundu-mukherjee-naskar model, Applied Mathematics and Nonlinear Sciences 4 (2019) 513.

25. Q. Zhou, H. Rezazadeh, A. Korkmaz, M. Eslami, M. Mirzazadeh, M. Rezazadeh, New optical solitary waves for unstable Schrödinger equation in nonlinear medium, Optica Applicata 49 (2019).

26. A.-H. Abdel-Aty, M. M. Khater, D. Baleanu, S. Abo-Dahab, J. Bouslimi, M. Omri, Oblique explicit wave solutions of the fractional biological population (bp) and equal width (ew) models, Advances in Difference Equations 2020 (2020) 1.

27. Y. Song et al., Nonlinear few-layer mxene-assisted all-optical wavelength conversion at telecommunication band, Advanced Optical Materials 7 (2019) 1801777.

28. B. Guan, S. Chen, Y. Liu, X. Wang, J. Zhao, Wave patterns of $(2+1)$-dimensional nonlinear heisenberg ferromagnetic spin chains in the semiclassical limit, Results in Physics 16 (2020) 102834 .

29. M. Latha, C. C. Vasanthi, An integrable model of $(2+1)-$ dimensional Heisenberg ferromagnetic spin chain and soliton excitations, Physica Scripta 89 (2014) 065204.

30. J. Sun, D. Nie, W. Deng, Fast algorithms for convolution quadrature of riemann-liouville fractional derivative, Applied Numerical Mathematics 145 (2019) 384.

31. J. Cresson, A. Szafrańska, Comments on various extensions of the riemann-liouville fractional derivatives: About the leibniz and chain rule properties, Communications in Nonlinear Science and Numerical Simulation 82 (2020) 104903.

32. S. Qureshi, A. Yusuf, Modeling chickenpox disease with fractional derivatives: From caputo to atanganabaleanu, Chaos, Solitons and Fractals 122 (2019) 111.

33. O. Brandibur, E. Kaslik, D. Mozyrska, M. Wyrwas, Stability of caputo-type fractional variable-order biquadratic difference equations, in: New Trends in Nonlinear Dynamics, Springer, 2020, pp. 295-303. 
34. P. V. S. Mascarenhas, R. M. de Moraes, A. L. B. Cavalcante, Using a shifted grünwald-letnikov scheme for the caputo derivative to study anomalous solute transport in porous medium, International Journal for Numerical and Analytical Methods in Geomechanics 43 (2019) 1956.

35. E. M. Mendes, G. H. Salgado, L. A. Aguirre, Numerical solution of caputo fractional differential equations with infinity memory effect at initial condition, Communications in Nonlinear Science and Numerical Simulation 69 (2019) 237.

36. A. A. Abdelhakim, Precise interpretation of the conformable fractional derivative, arXiv preprint arXiv:1805.02309 (2018).
37. M. Darvishi, M. Najafi, A. Wazwaz, New extended rational trigonometric methods and applications, Waves in Random and Complex Media 30 (2020) 5.

38. N. Mahak, G. Akram, Extension of rational sine-cosine and rational sinh-cosh techniques to extract solutions for the perturbed nlse with kerr law nonlinearity, The European Physical Journal Plus 134 (2019) 159.

39. N. Mahak, G. Akram, Exact solitary wave solutions by extended rational sine-cosine and extended rational sinh-cosh techniques, Physica Scripta 94 (2019) 115212. 